\title{
The Virus of Vagueness in Authorship
}

\author{
David Shaw
}

Published online: 5 July 2016

(C) Journal of Bioethical Inquiry Pty Ltd. 2016

Authorship is an essential component of assessing researchers' contributions to academia and science. But authorship lists are now recognized as a crude and vague way of communicating contributions because they are simply not very informative (Cutas and Shaw 2014). Generally, they inform readers about who made a substantial contribution to a research paper or project but provide no information regarding the extent or variety of that contribution (Shaw and Erren 2015). Because of this vagueness, readers often make assumptions about contributions based on the order in which authors are listed. But these assumptions often introduce further confusion because they are based on the fundamental assumption that all researchers use the same paradigms of authorship order. In fact, they do not, which makes the vagueness surrounding authorship even worse. Ambiguity concerning author input into published papers is recognized as a substantial problem within individual disciplines, but the problem is a particular issue in interdisciplinary publications, where any lack of contributorship statements will mean that the perceived contributions from each author will be dependent on the dominant paradigm used by each reader.

A thought experiment can illustrate the problem. Let's imagine three researchers from different fields named Robert Roberts, James Jameson, and Charles Charleston write an interdisciplinary research paper

D. Shaw $(\bowtie)$

Institute for Biomedical Ethics, University of Basel, Bernoullistrasse 28, 4056 Basel, Switzerland e-mail: david.shaw@unibas.ch together. The paper is read by many people, but we focus on three in particular: Ava, Anna, and Jana. They all enjoy the paper and are interested in collaborating with the person who had the idea for it. The order of authors given at the start of the paper is Charles, James, Robert.

Ava works in the medical faculty and assumes that Robert had the idea, because he is last author and thus senior. These assumptions are mistaken: he might not be senior, and even if he was, that doesn't mean it was his idea. Anna works in philosophy and assumes that Charles did the most work and it was probably his idea too, because in philosophy authors tend to be listed in diminishing order of contribution. Here too, there are two assumptions: that he did more work than the second author, Charles, and that the person who did most work also had the idea. Finally, Jane is a biologist who always uses the principle that authors are listed alphabetically. She therefore assumes that the same has happened here and emails Charles (who happens to be the corresponding author) to ask who did what.

All three readers are mistaken: it turns out that they all three authors contributed equally to the conceptualization and writing of the paper and that they decided on authorship order by randomization. In all these cases, a fundamental assumption about authorship attribution paradigms was made in addition to various other assumptions. And this is the problem with vagueness in authorship: vagueness about the system used to decide on author order, and about authors' specific contributions, seeds mistaken assumptions which further distort the intended message. 
Vagueness can also cause problems for authors who are attempting to agree on a fair ordering of authors on a paper. I once started writing a paper with a colleague, and we decided to ask another researcher to contribute to the paper. Just for convenience, my colleague added this third author after my name, turning me into the middle author. When we got to the point of submitting the article, we had a conversation about authorship. I said he should be middle and I last author because he contributed less than me (and because I work in an institute associated with a medical faculty so tend to apply that paradigm). However, he said he should be last author because he contributed less than me. Who was right? Objectively, it might make more sense to adopt the diminishing contribution model, as he suggested. But if the paper is to be published in a medical journal and almost everyone reading it will apply the medical paradigm (as I assumed), I will be penalized, and he will benefit, from the vagueness around our contributions and from the readers' consequent assumptions. Paradoxically, on an earlier paper we had written together with the same colleague and on which I thought he had contributed more, he was last author and did not claim that he should be middle author because I had done less.

Ironically, vagueness can actually help in resolving authorship order dispute in certain interdisciplinary teams. If a philosopher and a doctor collaborate on a paper, the philosopher might prefer to be first author and the doctor last author because of the differing interpretations of their respective disciplines and departments. Staff in the medical faculty will assume that the doctor was the main intellectual contributor, and philosophers will assume the opposite. Here, vagueness about contributions actually works in favour of both authors because vagueness generated by the differences between disciplines enables compromise that is to the benefit of both authors.

The problem of vagueness provides yet another reason why traditional authorship lists should be abandoned in favour of concise contributorship statements placed at the start of published research papers. The current system of putting any such statements at the end of papers while retaining author lists at the start is an unfortunate compromise which allows vagueness to influence decisions about collaborations, grants, and hiring. Given the importance attached to evidence in science, it is unfortunate that the virus of vagueness should still infect authorship to the extent that it does.

\section{References}

Cutas, D., and D. Shaw. 2014. Writers blocked: On the wrongs of research co-authorship and some possible strategies for improvement. Science and Engineering Ethics 21(5): 13151329.

Shaw, D., and T. Erren. 2015. 10 simple rules for protecting research integrity. PLOS Computational Biology 5. doi:10.1371/journal.pcbi.1004388. 